\title{
Tendencias del consumo de tabaco entre alumnos de nuevo ingreso a la Universidad Nacional Autónoma de México, 1989 a 1998
}

\author{
Raydel Valdés-Salgado, MSc, ${ }^{(1)}$ Juan Manuel Micher, MD, ${ }^{(2)}$ Luis Hernández,MD, ${ }^{(2)}$ \\ Melania Hernández, MD, ${ }^{(2)}$ Mauricio Hernández-Avila, MD, ScD.(1)
}

Valdés-Salgado R, Micher JM, Hernández L, Hernández M, Hernández-Avila M.

Tendencias del consumo de tabaco entre alumnos de nuevo ingreso a la Universidad Nacional Autónoma de México, 1989 a 1998. Salud Publica Mex 2002;44 supl 1:S44-S53. El texto completo en inglés de este artículo está disponible en: http://www.insp.mx/salud/index.html

\section{Resumen}

Objetivo. Describir el consumo de tabaco entre estudiantes de la U niversidad $\mathrm{N}$ acional Autónoma de México (UN AM) durante 1989 a 1998. Material y métodos Es un estudio de series de tiempo, hecho en el año 2000, en el cual se analiza una cohorte. Se calculó la prevalencia de consumo entre 103582 alumnos de 14 a 25 años, de nuevo ingreso a la UN AM y las prevalencias específicas por edad y sexo, así como la prevalencia de fumadores de 11 o más cigarros diarios. Se identificó a los mismos estudiantes en los dos momentos y se estimó la incidencia acumulada de consumo de tabaco durante el periodo de educación media superior. Se aplicó la prueba ji cuadrado de tendencias y se construyó un modelo de regresión logística para obtener razones de momios de prevalencia. Resultados Se observó un aumento en la prevalencia de consumo de tabaco. Particularmente se ha incrementado el consumo en las edades menores de 18 años y entre las mujeres, lo anterior es más evidente a partir de 1993. Los estudiantes de mejor situación económica muestran una mayor incidencia de inicio del consumo. D urante los años de enseñanza media superior, en general, la incidencia de inicio del consumo es de $12 \%$ para las mujeres y de $19 \%$ para los varones. A mayor edad, ser varón y al final del periodo la probabilidad de fumar aumenta. Conclusiones En la última década se observa una tendencia ascendente en el consumo de tabaco entre estudiantes. Es necesario instrumentar campañas antitabaco. El texto completo en inglés de este artículo está disponible en: http://www.insp.mx/salud/index.html

Palabras clave: tabaquismo; adolescencia; prevalencia; incidencia; México

\author{
Valdés-Salgado R, Micher JM, Hernández L, \\ Hernández M, Hernández-Avila M. \\ Trends in tobacco consumption \\ among new students in Universidad \\ Nacional Autonoma de Mexico, from 1989 to 1998. \\ Salud Publica Mex 2002;44 suppl 1:S44-S53. \\ The English version of this paper \\ is available at: http://www.insp.mx/salud/index.html
}

\begin{abstract}
A bstract
Objective. The purpose of this study was to describe the tobacco smoking patterns in students in the Universidad $\mathrm{N}$ acional Autonoma de Mexico system, (Mexico's N ational Autonomous U niversity, UN AM), between 1989 and 1998. Material and Methods. This is a time-series, cohort study conducted in the year 2000. The overall prevalence of smoking, age- and sex-specific prevalence rates, and the prevalence of subjects smoking 11 or more cigarettes daily, were estimated for 103582 new students aged 14 to 25 years. The same subjects were contacted for a second time, to estimate the cumulative incidence of smoking during the high school period. Associations were tested using a chisquared test for trends, and prevalence odds ratios were obtained using a logistic regression model. Results. The overall prevalence of smoking increased during the study perio d, mainly after 1993 among younger and female subjects. A higher smoking incidence was found among students of higher economic level. During the high school years, the incidence of smoking initiation was $12 \%$ for females and $19 \%$ for males. The probability of smoking increased with older age, male gender, and at the final years of the study period. Conclusions. In the last decade, evident changes in young students' smoking patterns have occurred. Antitobacco campaigns targeting young people should be implemented. The English version of this paper is available at: http://www.insp.mx/salud/index.html
\end{abstract}

Key words: smoking; adolescence; prevalence; incidence; Mexico

(1) Centro de Investigación en Salud Poblacional, Instituto Nacional de Salud Pública, Cuernavaca, Morelos, México.

(2) Dirección General de Servicios Médicos, Universidad N acional Autónoma de México, México, D.F., México.

Fecha de recibido: 30 de mayo de 2001 • Fecha de aprobado: 5 de noviembre de 2001

Solicitud de sobretiros: Maestro Raydel Valdés Salgado. Instituto N acional de Salud Pública. Avenida Universidad 655, colonia Santa María A huacatitlán, 62508 Cuernavaca, Morelos, México. Correo electrónico: rayvs@correo.insp.mx 
$\mathrm{D}$ esde el decenio de 1970 a escala mundial se describe un aumento en el consumo de tabaco entre los adolescentes de los países de ingreso medio y bajo, aunque actualmente esa tendencia se ha desacelerado desde inicio de los años 90.1,2 Actualmente, los niveles de consumo reportados ${ }^{3}$ entre los adolescentes y jóvenes de los países de América Latina varían desde 4.4 hasta $40.7 \%$. En México es importante conocer las tendencias actuales del consumo para diseñar intervenciones efectivas que desalienten a los adolescentes para fumar.

En países desarrollados como los Estados Unidos de América (EUA) se dio un descenso de la prevalencia en el consumo de tabaco entre adolescentes de uno $\mathrm{u}$ otro sexo desde mediados de los años 70 hasta principios de los $90 .{ }^{4}$ Sin embargo, las últimas investigaciones muestran consistentemente un incremento en el consumo entre estudiantes de educación media superior. Por ejemplo, en 1989 la prevalencia de tabaquismo era de 15.3 y $16.0 \%$ para mujeres y hombres respectivamente, mientras que para 1995 aumentó a $34.3 \%$ en las mujeres y a $35.4 \%$ para los hombres. ${ }^{5-10}$ Otro fenómeno en ascenso es el consumo de otras formas de tabaco sin humo. ${ }^{9,11,12}$

\section{Los inicios del consumo en la adolescencia. Reportes de países desarrollados}

La adolescencia temprana -menores de 15 años- es el periodo en el que generalmente las personas intentan fumar por primera vez, de ahí que algunos autores propongan considerar el fumar cigarrillos como una enfermedad pediátrica. ${ }^{13}$ En los EUA se ha reportado que el riesgo de fumar es mayor cuando coinciden otros factores como padres que fuman, o pertenencia a hogares de bajos ingresos o bajo nivel educativo. ${ }^{14}$

Entre los factores sociales que más frecuentemente aparecen asociados con el inicio del tabaquismo en la adolescencia está la presión psicosocial de los amigos, que puede conducir a la experimentación temprana del consumo. Es común que por causa del desconocimiento los adolescentes sobrestimen la prevalencia de fumadores entre la población en general, al mismo tiempo que desestiman la posibilidad de llegar a convertirse en fumadores habituales. Percepciones erróneas como las anteriores se explican en parte por la publicidad intencionalmente diseñada por las tabacaleras para atraer gente joven. ${ }^{15}$

Una de las creencias que más se relaciona con la iniciación en el consumo es que fumar se asocia positivamente con tener una imagen social deseada, o como un facilitador para establecer relaciones. ${ }^{16}$ En el caso de las muchachas, el consumo de cigarrillos también puede ser visto como un medio para mantener el control de peso y como una conducta glamorosa. También, algunos adolescentes fuman para reafirmar el paso de la niñez a la adultez. ${ }^{14,17}$

Los adolescentes subestiman la posibilidad de volverse adictos a la nicotina. Se ha comunicado que entre los adolescentes varones que fuman, la posibilidad de que cambien de marca hacia una de más alto contenido de nicotina es dos veces mayor a que el cambio sea en sentido opuesto. ${ }^{18}$

\section{Antecedentes en México de estudios del consumo de tabaco en estudiantes}

Los trabajos previos son estudios transversales, en muestras seleccionadas de la población estudiantil , la mayor parte de los cuales se han realizado en el Distrito Federal, aunque en 1991 se realizó una encuesta nacional. $^{19}$

En 1980 se reportó que el consumo de tabaco alguna vez en la vida entre la población estudiantil de 14 a 18 años fue de $55.8 \%$ para los varones, mientras que para las mujeres el valor fue de $37.9 \%$. Para la muestra en general el consumo de tabaco, al menos ocasionalmente, fue de $46.9 \% .{ }^{20}$ En el mismo año, otro estudio realizado entre alumnos de nuevo ingreso a algunas facultades de la UNAM y a la Escuela Nacional de Estudios Profesionales de Cuautitlán* reportan una prevalencia de fumadores habituales -no se dan detalles de su definición- de 36.3\%. Entre los hombres la cifra fue $39.7 \%$, y entre las mujeres $27.6 \%$. Para los estudiantes de la UNAM, las prevalencias de consumo de tabaco en hombres y mujeres fueron 40.0 y $30.1 \%$, respectivamente.

En el curso 1987-1988 se reporta, sin dar más detalles al respecto, una prevalencia de consumo de tabaco de $13.1 \%$ entre alumnos de nuevo ingreso a la UNAM. De los fumadores identificados, $40 \%$ se inició en el hábito antes de los 15 años. ${ }^{\ddagger}$

En 1991 se realizó la Encuesta Nacional sobre el Uso de Drogas en la Comunidad Escolar ${ }^{19}$ entre es-

\footnotetext{
* Casillas LE, Benavides L. Epidemiología del tabaquismo y del alcoholismo entre los universitarios. S/f: 138-155. Universidad Nacional Autónoma de México. Documento interno.

‡ Hernández LE, Domínguez JA, Ramírez M, González R. Uso de sustancias tóxicas en población estudiantil de primer ingreso a la UNAM ciclo escolar 1987-1988. Resultados preliminares. S/f: 7682. Documento interno.
} 
tudiantes de educación media y media superior, y reportó $29.4 \%$ de prevalencia de estudiantes que han fumado o masticado tabaco alguna vez en su vida. Entre los varones la cifra llegó a $38.1 \%$ y para las mujeres fue de $20.2 \%$.

En 1993 y 1997 se realizaron encuestas como la anterior, pero sólo en el Distrito Federal. ${ }^{21,22}$ En la primera de ellas se reporta consumo de tabaco alguna vez en $48.0 \%$ de los estudiantes de nivel medio y medio superior. Entre los varones la cifra fue de $55.7 \%$ y para las mujeres de $41.2 \%$. Cuatro años más tarde al preguntar ihas fumado tabaco alguna vez en tu vida? la prevalencia total se elevó hasta $55.4 \%$, llegando a $58.6 \%$ entre los varones y para las mujeres también se incrementó y el valor llegó a 52.3\%.

La Encuesta Nacional de Salud ${ }^{23}$ refirió una prevalencia de fumadores actuales de $6.8 \%$ en el grupo de 18-20 años. Aunque no han sido orientadas a la población estudiantil específicamente, también están las tres aplicaciones de la Encuesta Nacional de Adicciones (ENA, 1988, 1993, 1998). ${ }^{24-26}$ En la más reciente, se reporta para el grupo 12-17 años una prevalencia de consumo de $7.4 \%$ para las mujeres y de $16.0 \%$ para los varones..$^{26}$ En las tres ENA realizadas, la pregunta de la que se obtuvo la prevalencia fue ¿Ha fumado tabaco alguna vez en la vida?

Si bien los antecedentes descritos son referencias valiosas, no es menos cierto que las cifras cambiantes y generalmente altas que ellos muestran quizá no sólo reflejan la magnitud y evolución del fenómeno en sí, sino también las diversas formas en que éste se ha medido a partir de diferentes definiciones conceptuales y operacionales. También, al tratarse de estudios aplicados en poblaciones heterogéneas, los intentos por hacer comparaciones se hacen difíciles, y más aún, riesgosos.

$\mathrm{Al}$ inicio de cada curso escolar la UNAM aplica a los nuevos alumnos un cuestionario médico y de estilos de vida junto a sus exámenes de ingreso al nivel medio superior y superior. Entre otros temas se recoge información sobre consumo de tabaco. Estos cuestionarios ofrecen una buena oportunidad, por su periodicidad y por tratarse de cohortes sucesivas de una misma población blanco, para acercarnos al comportamiento de la prevalencia de consumo de tabaco entre jóvenes de edades específicas al entrar a la educación media superior y superior. El objeto de este trabajo es describir cómo se ha comportado el consumo de tabaco entre los adolescentes y jóvenes que estudian en la UNAM; para ello analizamos información del periodo 1989-1998. En México no hay referencias previas de estudios en población estudiantil que describan el consumo durante un lapso.

\section{Material y métodos}

La población que se estudió fueron 103582 alumnos de 14 a 25 años; de ellos, 52016 hombres y 51553 mujeres. Se consideraron dos aspectos como criterios de inclusión. Primero, se definieron dos grupos según niveles educativos: 42488 estudiantes (53.8\% hombres y $46.2 \%$ mujeres) de nuevo ingreso a la educación media superior y 61094 estudiantes $(47.8 \%$ hombres y 52.2 mujeres) que ingresan a la educación superior. El segundo criterio es la procedencia de las escuelas que aparecen en cada uno de los años de estudio, lo cual permitió la comparación. No se manejó ningún dato que pudiera ser revelador de la identidad de los alumnos.

Para el grupo de la enseñanza media superior se dispuso de información de los planteles 1 al 5 de la Escuela Nacional Preparatoria. La información del grupo de educación superior se obtuvo de los nuevos ingresos a las facultades de Arquitectura, Química, Derecho, Economía, Filosofía y Letras, Ingeniería, Medicina, Medicina Veterinaria y Psicología. También se contó con información de las Escuelas Nacionales de Artes Plásticas, Enfermería, Música y Trabajo Social, todas pertenecientes a la UNAM.

Para hacer comparables las poblaciones de los diferentes años se realizó una estandarización directa tomando como referencia la estructura por sexo y edad del año 1989.

Para caracterizar el consumo de tabaco se calcularon prevalencias de consumo generales y específicas ajustadas por edad y por sexo para cada año analizado. También se analizó la prevalencia de estudiantes que fuman diariamente y el consumo de 11 o más cigarrillos por día, lo cual se puede considerar como un indicador de adicción temprana.

La variable dependiente de este trabajo se conformó a partir de las respuestas a la pregunta Cuando fumas en un día cualquiera, ¿cuántos cigarros consumes? Para toda respuesta que se refiriera a un determinado número de cigarrillos (cuadro I) se consideró como que esa persona consumía tabaco y, si no seleccionó ninguna opción se consideró como no consumidor. Finalmente, tuvimos una variable dicotómica donde el 1 señala la presencia del atributo, en este caso el consumo de tabaco y con el 0 se designa la ausencia del mismo.

La incidencia acumulada de consumo de tabaco se calculó ${ }^{27}$ dividiendo el número de casos nuevos que reportaron que sí consumían al entrar a la universidad, pero que en la primera aplicación aún no eran fumadores, entre el total de estudiantes que en 1989 ingresó a la educación media superior a los cuales se 


\section{Cuadro I \\ Pregunta que da origen A NUESTRA VARIABLE DEPENDIENTE}

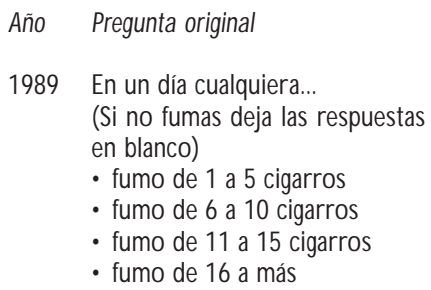

1989 En un día cualquiera... (Si no fumas deja las respuestas en blanco)

- fumo de 1 a 5 cigarros

- fumo de 6 a 10 cigarros

- fumo de 11 a 15 cigarros

- fumo de 16 a más

Se creó una variable dicotómica. Si el alumno(a) seleccionó al menos una opción, entonces asignamos 1 y se consideraba que consumía tabaco. Si no, se asignaba 0 y se consideró que no era consumidor(a).

1990 A continuación aparecen algunas preguntas respecto al uso de tabaco: escoge las opciones que más tengan que ver contigo.

(Si no fumas deja las respuestas en blanco)

- fumo de 1 a 5 cigarros

- fumo de 6 a 10 cigarros

- fumo de 11 a 15 cigarros

- fumo de 16 a más

1992 Cuando fumas, ¿cuántos cigarros consumes por día?

- 1 cigarro

- de 2 a 10

- de 11 a 20

- de 21 a 30

Se creó una variable dicotómica. Si el alumno(a) seleccionó al menos una opción, entonces asignamos 1 y se consideraba que consumía tabaco. Si no, se asignaba 0 y se consideró que no era consumi$\operatorname{dor}(a)$.

Se creó una variable dicotómica. Si el alumno(a) seleccionó al menos una opción, entonces asignamos 1 y se consideraba que consumía tabaco. $\mathrm{Si}$ no, se asignaba 0 y se consideró que no era consumi$\operatorname{dor}(a)$.

1993 Cuando fumas, ¿cuántos cigarros consumes por día?

- 1 cigarro

- de 2 a 10

- de 11 a 20

- de 21 a 30

Se creó una variable dicotómica. Si el alumno(a) seleccionó al menos una opción, entonces asignamos 1 y se consideraba que consumía tabaco. Si no, se asignaba 0 y se consideró que no era un consumi$\operatorname{dor}(a)$. ca. Si el alumno(a) seleccionó
1996 Cuando fumas, ¿cuántos cigarros consumes por día?

- 1

- 2 a 10

- 11 a 20

- 21 a 30

- 310 más

Se creó una variable dicotómial menos una opción, entonces asignamos 1 y se consideraba que consumía tabaco. $\mathrm{Si}$ no, se asignaba 0 y se consideró que no era consumi$\operatorname{dor}(a)$.

Con la creación de la variable dicotómica se pudo comparar la información obtenida de cuestionarios de diferente formato y así estimar la prevalencia de consumo de tabaco.También se pudo determinar para cada año el consumo diario de 11 cigarrillos o más y distinguir a los fumadores en tres categorías: los que fuman menos de 10 cigarros al día; los que fuman entre 11 y 20; los que fuman más de 21 diariamente.

Fuente:EM PI-U niversidad N acional Autónoma de México, 1989, 1990,1992, 1993,1996

les pudo dar seguimiento. La comparación de la información recogida en los dos momentos se hizo a través de la variable número de cuenta, y en cada caso se confirmó que se trataba de la misma persona verificando si la edad y el sexo correspondían.
De la cohorte de alumnos que ingresó en 1989 a la educación media superior $(n=7256)$ se identificaron 2438 estudiantes (33.6\%) a su ingreso a la universidad (1 368 mujeres y 1070 hombres) y se compararon sus respuestas a las preguntas sobre consumo de tabaco en ambos momentos. Lo que impidió un seguimiento más extenso fue el no disponer de la información referida a 1994 y 1995, la deserción escolar y, en menor medida, los casos que no recuperó el sistema de cómputo.

Los resultados que involucran el nivel socioeconómico se basan en los datos del año 1989, a partir de los cuales se distinguen cuatro niveles. El más bajo corresponde a los alumnos procedentes de familias que mensualmente recibían ingresos que no superaban los dos salarios mínimos de aquel año; le siguen alumnos cuyas familias recibían hasta cuatro salarios mínimos, después los de hasta seis salarios mínimos y los del nivel más alto recibían en sus hogares siete salarios mínimos o más.

Se calculó la prueba de ji cuadrado para ver la diferencia de prevalencias. Se utilizaron modelos de regresión logística, para obtener razones de momios de prevalencias, con intervalos de confianza, IC, al 95\%. El procesamiento de la información se realizó con el paquete estadístico SPSS 9.0.

\section{Resultados}

La figura 1 muestra el primer resultado derivado de nuestro estudio, el cual fue que a lo largo de los años analizados se incrementó significativamente $(p<0.001)$ la prevalencia de fumadores en ambos grupos de edades, lo cual describe una tendencia ascendente del consumo. En los cuadros II y III se presentan las pre-

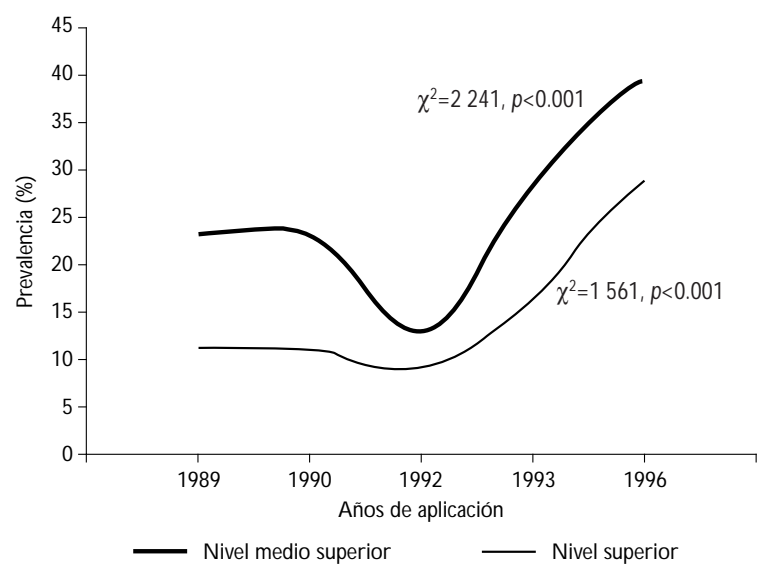

Figura 1. Prevalencia ajustada de consumo de tabaco EN ALUMNOS DE NUEVO INGRESO A LA UNIVERSIDAD Nacional Autónoma de México 
valencias ajustadas específicas para cada edad en hombres y mujeres, y se aprecia que a partir del año 1993 los valores se elevan bruscamente. En ese año, de los alumnos que ingresan a la educación media superior IC 95\% 16.3\% (16.1-16.5,) reconoce que consume taba$\mathrm{CO}$, mientras que para los que inician la educación superior $28.4 \%$ (IC 95\% 28.1; 28.7,) acepta que son consumidores de tabaco.

\section{Evolución de la prevalencia de consumo de tabaco entre adolescentes y jóvenes}

Entre los varones del grupo 14-18 años el consumo creció más del doble entre el inicio y el final del periodo de años comparados -de 14.6\% (IC 95\% 14.5; 14.8,) en 1989 a 33.1\% (IC 95\% 32.8; 33.4,) en $1996(p<0.001)$. Analizando las prevalencias específicas por edad, aparece que en ese lapso los mayores incrementos ocurrieron en las edades 14 y 15 años, donde los valores se triplican entre el inicio y el final del periodo. En las edades 16, 17 y 18 años también se aprecia un aumen-

\section{Cuadro II}

Prevalencias ajustadas, generales y específicas POR EDAD, DE CONSUMO DE TABACO ENTRE ALUMNOS DE NUEVO INGRESO A LA EDUCACIÓN MEDIA SUPERIOR. Universidad Nacional Autónoma de México, México

Prevalencia gene-

$1989199019921993 \quad 1996 \quad$ Signif.

ral de consumo $\quad$ Hombres $14.6 \quad 14.2 \quad 11.9 \quad 21.2 \quad 33.1 \quad p<0.001$

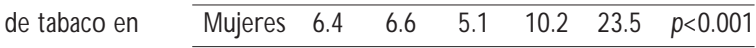

$\begin{array}{lllllllll} & \text { cada año } & \text { Total } & 11.0 & 10.8 & 8.9 & 16.3 & 28.8 & p<0.001\end{array}$

Prevalencias específicas de consumo ajustadas por edad

\begin{tabular}{llrrrrr} 
& Sexo & 14 años & 15 años & 16 años & 17 años & 18 años \\
1989 & Hombres & 5.5 & 10.6 & 21.0 & 22.4 & 38.1 \\
\hline & Mujeres & 3.9 & 5.7 & 8.4 & 15.3 & 11.8 \\
\hline 1990 & Hombres & 4.7 & 10.8 & 19.4 & 28.8 & 24.5 \\
\hline & Mujeres & 2.4 & 6.1 & 11.9 & 10.3 & 12.8 \\
\hline 1992 & Hombres & 4.8 & 10.7 & 15.7 & 14.3 & 19.5 \\
\hline & Mujeres & 3.5 & 7.7 & 12.1 & 12.3 & 11.9 \\
\hline 1993 & Hombres & 10.3 & 18.3 & 26.6 & 28.2 & 37.4 \\
\hline & Mujeres & 3.6 & 9.6 & 20.9 & 14.3 & 9.4 \\
\hline 1996 & Hombres & 15.2 & 33.1 & 36.6 & 36.3 & 36.8 \\
\hline & Mujeres & 13.9 & 23.9 & 28.2 & 23.2 & 13.8
\end{tabular}

Fuente: estimaciones propias con base en datos de la Universidad $\mathrm{N}$ acional Autónoma de México.

Todas las diferencias de prevalencia específicas para cada edad (14-18 años) observadas en el periodo 1989-1996 son estadísticamente significativas $(p<0.001)$ to, puesto que los valores crecieron hasta duplicarse $(p<0.001)$, (cuadro II).

Entre las mujeres de 14-18 años los incrementos en el consumo son claros. Al inicio del periodo, en general, se observan valores de prevalencia relativamente bajos, $6.4 \%(6.3 ; 6.5)$ en 1989 , pero que van creciendo hasta casi cuadruplicarse, $23.5 \%(23.3 ; 23.7)$ en el último año comparado, 1996 ( $p<0.001)$. El análisis de las prevalencias específicas muestra que en las edades 14 , 15 y 16 años ocurren incrementos a lo largo del periodo, en los cuales las cifras iniciales se triplican y aun, se cuadruplican para la edad 15 años $(p<0.001)$, (cuadro II).

En el grupo 18-25 años también se observó un incremento en el consumo de tabaco en uno $u$ otro sexo, ya que en general la prevalencia aumentó de $23.2 \%$ (IC $95 \% 22.9 ; 23.5$,) en 1989 a $39.5 \%$ (IC $95 \% 39.1 ; 39.9$,) en 1996. Para los varones que entraron a la universidad, el consumo creció desde 28.3\% (IC 95\% 28.0; 28.6,) en

\section{Cuadro III \\ Prevalencias ajustadas, generales y específicas POR EDAD, DE CONSUMO DE TABACO ENTRE ALUMNOS DE NUEVO INGRESO A LA EDUCACIÓN SUPERIOR, Universidad Nacional Autónoma de México, México}

Prevalencia gene-

19891990199219931996 Signif.

ral de consumo $\quad$ Hombres $28.3 \quad 27.1 \quad 13.0 \quad 33.2 \quad 42.4 \quad p<0.001$ \begin{tabular}{lllllllll}
\cline { 2 - 7 } de tabaco en $\quad$ Mujeres & 17.6 & 17.5 & 12.6 & 22.9 & 36.1 & $p<0.001$ \\
\cline { 2 - 7 } & & &
\end{tabular} \begin{tabular}{llllllll}
\cline { 2 - 6 } cada año & Total & 23.2 & 22.6 & 12.8 & 28.4 & 39.5 & $p<0.001$
\end{tabular}

Prevalencias específicas de consumo ajustadas por edad

$\begin{array}{lllllllll}\text { Sexo } & 18 & 19 & 20 & 21 & 22 & 23 & 24 & 25\end{array}$ años años años años años años años años

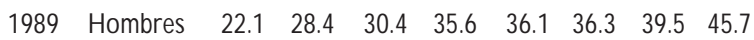

\begin{tabular}{cllllllll}
\hline Mujeres & 14.4 & 20.2 & 20.2 & 20.4 & 24.3 & 19.2 & 21.1 & 25.0
\end{tabular}

\begin{tabular}{llllllllll}
\hline 1990 & Hombres & 19.2 & 28.4 & 32.9 & 34.4 & 30.5 & 37.9 & 31.3 & 33.7
\end{tabular}

\begin{tabular}{lllllllll} 
Mujeres & 14.0 & 20.9 & 21.8 & 17.8 & 16.5 & 16.9 & 18.8 & 19.9 \\
\hline
\end{tabular}

\begin{tabular}{llllllllll}
\hline 1992 & Hombres & 8.4 & 13.6 & 15.0 & 17.1 & 19.3 & 15.4 & 13.4 & 17.9
\end{tabular}

$\begin{array}{lllllllll}\text { Mujeres } & 11.3 & 13.0 & 17.0 & 11.6 & 12.5 & 10.8 & 12.1 & 6.4\end{array}$

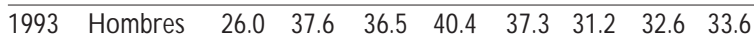

$\begin{array}{lllllllll}\text { Mujeres } & 21.7 & 26.4 & 23.0 & 21.5 & 22.4 & 14.2 & 18.9 & 9.7\end{array}$

\begin{tabular}{lllllllllll}
\hline 1996 & Hombres & 41.1 & 42.6 & 39.9 & 44.4 & 38.9 & 53.1 & 45.4 & 36.8
\end{tabular}

$\begin{array}{lllllllll}\text { Mujeres } & 41.3 & 35.8 & 29.5 & 27.1 & 29.6 & 23.2 & 23.7 & 25.3\end{array}$

Fuente: estimaciones propias en base a datos de la Universidad $\mathrm{N}$ acional Autónoma de México.

Todas las diferencias de prevalencia específicas para cada edad (18-25 años) observadas en el periodo 1989-1996 son estadísticamente significativas $(p<0.001)$. Para la edad 24 años en las mujeres, las diferencias son estadísticamente significativas con $p<0.05$ 
1989 hasta $42.4 \%$ (IC 95\% 41.9; 42.8,) al final del periodo. $(p<0.001)$. Analizando las prevalencias específicas por edad aparece que en general los valores se incrementan durante el lapso, particularmente para las edades inferiores a 21 años $(p<0.001)$, (cuadro III).

Entre las mujeres de 18-25 años el incremento en el consumo se duplica, de $17.6 \%$ (IC 95\%, 17.4; 17.8) en 1989 a 36.1\% (35.8; 36.5, IC 95\%) en 1996. El análisis específico por edad muestra que en los 18 años ocurre el crecimiento del consumo más sobresaliente, ya que entre 1989 y 1996 los valores se triplican. ( $p<0.0$ (cuadro III).

Se comparó el consumo diario de cigarros entre el inicio y el final del periodo. Las prevalencias ajustadas del grupo de edad 18-25 años muestran que entre los varones se mantuvo estable en $13.7 \%$ (IC 95\%, 13.6; 13.8). Sin embargo, sí hubo un incremento entre las mujeres, ya que al inicio del periodo la prevalencia de consumo diario era de 7.6\% (IC 95\% 7.5; 7.7,) y para 1996 había crecido hasta 9.8\% (IC 95\% 9.7; 9.9,).

El consumo de 11 o más cigarrillos al día, que pudiera tomarse como un indicador de adicción, se mantuvo con una prevalencia baja, según la información disponible de los años 1990, 1992, 1993, 1996 y 1998, aunque sí se observa un incremento en el grupo 18-25 años, sobre todo entre las mujeres. Por ejemplo, en 1990 los valores observados en hombres y mujeres fueron 3.11 (IC 95\% 3.08; 3.15,) y 1.41 (IC 95\% 1.38; 1.43,), mientras que para 1998 fueron 4.06 (IC 95\% 4.02, 4.11,) y 3.81 (IC 95\% 3.78; 3.85,) respectivamente. Entre los alumnos que ingresaron a la educación media superior los valores se mantienen inferiores a $0.5 \%$ todos los años.

\section{Incidencia del inicio de consumo durante los años de educación media superior}

De las mujeres cuyas respuestas se analizaron en los dos momentos, $84.5 \%$ se mantenía sin fumar al entrar a la universidad; $12.2 \%$ se inició en el consumo, lo cual constituye la incidencia acumulada durante los años de educación media superior; $2.1 \%$ abandonó el consumo y $1.2 \%$ se había mantenido consumiendo tabaco desde la primera aplicación.

Entre los varones a los que se les dio seguimiento, para cuando entraron a la universidad, $72 \%$ se mantenía sin fumar; $19.4 \%$ se inició en el consumo, lo cual constituye la incidencia acumulada durante ese periodo, mientras que $4 \%$ abandonó el hábito y $4.6 \%$ se había mantenido consumiendo tabaco desde la primera aplicación.

Se tomó al grupo de estudiantes que concluyeron la educación media superior en tres años e inmediata- mente ingresaron a la universidad (periodo académico 1989-1992) y se analizó la incidencia acumulada del consumo de tabaco según el nivel socioeconómico al que pertenecían los alumnos en 1989.

En general se observa un incremento del número de adolescentes que comienzan a fumar durante sus tres años de educación media superior. La incidencia ajustada por la edad fue de $10.0 \%$ (IC 95\% 9.92; 10.12,) y $8.1 \%$ (IC 95\% 8.04; 8.29,) para hombres y mujeres, respectivamente. Las incidencias más altas se observan para ambos sexos entre los estudiantes de mejor nivel socioeconómico, 15\% (IC 95\% 14.7; 15.04,) para los varones y $10 \%$ (IC 95\% 9.96; 10.17,) para las mujeres.

\section{Variables relacionadas con el consumo de tabaco en adolescentes y jóvenes estudiantes}

Se observa que conforme aumenta la edad de los estudiantes, en los años del final del periodo estudiado y la condición de ser varón, son factores que incrementan la probabilidad de consumir tabaco, según la razón de momios de prevalencias ajustada $(R M P)$ en el modelo de regresión logística (cuadro IV). En los adolescentes de 15 años la RMP es de 1.74, (IC 95\% de 1.53; 1.97). Para la edad 16 años la RMP es de 2.71 (IC 95\% de $2.37 ; 3.10$ ); para los 17 años la RMP es de 3.20 (IC de $95 \%$ de $2.69 ; 3.67)$; para 18 años la $R M P$ es de 3.25 (IC 95\% de $2.87 ; 3.68)$. Para la edad 19 años la RMP es de 4.28, (IC 95\% de 3.77; 4.86); para los 20 años la RMP es de 4.91 (IC 95\% de 4.31; 5.59). Para los estudiantes de 21 años la RMP es de 5.33 (IC de 95\% de 4.64; 6.11), para los de 22 la RMP es de 5.12 (IC 95\% de 4.43; 5.93). y para los de 23 la RMP es de 5.03 (IC 95\% de 4.29; 5.90). Finalmente, para los de 24 años la $R M P$ es de 4.81 (IC $95 \%$ de $4.04 ; 5.74$ ) y para los de 25 años la $R M P$ es de 5.44 (IC de $95 \%$ de $4.49 ; 6.60$ ). Lo antes descrito muestra una tendencia significativa $(p<0.001)$ con respecto a la edad y el consumo de tabaco.

También encontramos una tendencia significativa $(p<0.001)$ respecto al consumo de tabaco y el transcurso de los años analizados. En la medida en que avanzó el lapso estudiado, encontramos un incremento en las $R M P$, especialmente al final del periodo. En el año 1990 la RMP es de 0.95 (IC 95\% de 0.90; 1.00) y en 1992 la RMP es de 0.55 (IC 95\% de 0.52; 0.58). Pero en los dos últimos años el incremento es considerable, pues en 1993 la RMP es de 1.36 (IC 95\% de $1.29 ; 1.43$ ) y en 1996 la RMP es de 2.51 (IC 95\% de 2.39; 2.64).

$\mathrm{Al}$ analizar por género también encontramos resultados significativos $(p<0.001)$. La condición de ser varón tiene una RMP de 1.60 (IC 95\% de 1.55; 1.65). 


\section{Cuadro IV \\ Factores relacionados con el Consumo de tabaco EN ESTUDIANTES DE NUEVO INGRESO A LA UNIVERSIDAD Nacional Autónoma de México, México}

\begin{tabular}{|c|c|c|c|c|c|c|c|c|}
\hline \multirow{3}{*}{$\begin{array}{l}\text { Características } \\
\text { Edad en } \\
\text { años }\end{array}$} & \multicolumn{4}{|c|}{ Consumo de tabaco } & \multirow[t]{3}{*}{ RMP } & \multirow[t]{3}{*}{$\operatorname{IC}(95 \%)$} & \multirow[t]{3}{*}{ RMP* } & \multirow[t]{3}{*}{$I C(95 \%)$} \\
\hline & & & & $\sqrt{0}$ & & & & \\
\hline & Núm. & $\%$ & Núm. & $\%$ & & & & \\
\hline 14 & 305 & 7.50 & 3764 & 92.50 & 1.0 & & 1.0 & \\
\hline 15 & 3131 & 12.30 & 22325 & 87.70 & 1.73 & $1.53-1.96$ & 1.74 & $1.53-1.98$ \\
\hline 16 & 1478 & 17.54 & 6950 & 82.46 & 2.62 & $2.30-2.99$ & 2.72 & $2.38-3.10$ \\
\hline 17 & 1271 & 20.05 & 5067 & 79.95 & 3.10 & $2.71-3.53$ & 3.21 & $2.80-3.68$ \\
\hline 18 & 4958 & 20.48 & 19247 & 79.52 & 3.18 & $2.82-3.60$ & 3.26 & $2.90-3.70$ \\
\hline 19 & 3882 & 25.32 & 11447 & 74.68 & 4.20 & $3.70-4.73$ & 4.29 & $3.78-4.86$ \\
\hline 20 & 2277 & 27.73 & 5934 & 72.27 & 4.74 & $4.20-5.40$ & 4.91 & $4.31-5.60$ \\
\hline 21 & 1377 & 29.89 & 3230 & 70.11 & 5.26 & $4.60-6.00$ & 5.33 & $4.65-6.12$ \\
\hline 22 & 868 & 28.80 & 2146 & 71.20 & 4.99 & $4.34-5.75$ & 5.12 & $4.43-5.93$ \\
\hline 23 & 537 & 29.33 & 1294 & 70.67 & 5.12 & $4.40-5.97$ & 5.03 & $4.29-5.90$ \\
\hline 24 & 348 & 28.11 & 890 & 71.89 & 4.83 & $4.07-5.72$ & 4.81 & $4.04-5.75$ \\
\hline 25 & 257 & 30.02 & 599 & 69.98 & 5.29 & $4.40-6.38$ & 5.44 & $4.49-6.60$ \\
\hline \multicolumn{9}{|l|}{ Año } \\
\hline 1989 & 3015 & 17.82 & 13906 & 82.18 & 1.0 & & 1.0 & \\
\hline 1990 & 3326 & 17.79 & 15368 & 82.21 & 0.99 & $0.95-1.05$ & 0.95 & $0.90-1.00$ \\
\hline 1992 & 2959 & 10.62 & 24915 & 89.38 & 0.55 & $0.52-0.58$ & 0.55 & $0.53-0.59$ \\
\hline 1993 & 5129 & 23.54 & 16660 & 76.46 & 1.42 & $1.35-1.49$ & 1.36 & $1.30-1.44$ \\
\hline 1996 & 6260 & 34.20 & 12044 & 65.80 & 2.40 & $2.28-2.52$ & 2.51 & $2.39-2.65$ \\
\hline \multicolumn{9}{|l|}{ Sexo } \\
\hline Mujer & 8175 & 16.05 & 42753 & 83.95 & 1.0 & & 1.0 & \\
\hline Hombre & 12155 & 23.65 & 39234 & 76.35 & 1.62 & 1.67 & 1.60 & $1.55-1.65$ \\
\hline
\end{tabular}

RMP*: Razón de momios de prevalencia ajustada por las otras variables del modelo

\section{Discusión}

Los principales hallazgos de este trabajo son una tendencia significativa al aumento en la proporción de adolescentes y jóvenes que consumen tabaco en el periodo 1989-1996, particularmente en el grupo de menores de 18 años y entre las mujeres, lo cual se observa al analizar la evolución de las prevalencias específicas por edad; la incidencia de consumo durante la educación media superior es alta para ambos sexos, sobre todo entre los estudiantes de mejor posición socioeconómica, y un incremento en la prevalencia de los que consumen 11 o más cigarros al día. Las probabilidades de consumir tabaco se incrementan con la edad, mientras más avanzaba la década de los 90 respecto a 1989, y entre los varones respecto de las mujeres.

Es preocupante el incremento observado en el consumo en edades inferiores al mínimo legalmente establecido para poder adquirir tabaco en México, 18 años. Por lo tanto, además del problema de salud que representa ese consumo creciente, los datos ponen de manifiesto otras fallas. Evidentemente, las principales son la relajación en el cumplimiento de las actuales regulaciones a la venta de tabaco, ${ }^{28}$ la tolerancia social al consumo de cigarros e, indiscutiblemente, la efectividad de la publicidad de las tabacaleras. Quizá, también el comercio de contrabando a bajos precios.

Uno de los logros de la lucha contra el tabaco en México son las modificaciones hechas a la Ley General de Salud, que prohíbe fumar en los edificios públicos del Gobierno Federal. ${ }^{29}$ Para lograr revertir la tendencia descrita en los estudiantes, esfuerzos como el anterior deben coordinarse en todos los lugares públicos, y en especial en las escuelas, y también promover una cultura antitabaco en la comunidad, lo cual ya ha mostrado su eficacia, sobre todo en el ámbito escolar. $^{30-31}$

La incorporación de las mujeres es otro factor que ha incidido en el incremento general del consumo. En el patrón de prevalencias específicas por edad se ve claramente ese aumento de las estudiantes fumadoras, por lo tanto una de las líneas de investigacion que debe incorporarse en el futuro es la del enfoque de género, para entender mejor esta tendencia y cambiarla. Uno de los principales retos es reconceptualizar el cigarro como algo exclusivamente dañino para la salud y derribar el mito alimentado por las tabacaleras de que su consumo es un icono de glamour, independencia y éxito en la vida. Se ha señalado ${ }^{32}$ que cuando se emplean mensajes de salud para confrontar la promoción del tabaco, esto tiene desde el principio algún efecto entre las mujeres adultas jóvenes, pero no tanto entre las adolescentes. Sin embargo, cuando se trata de campañas prolongadas de contra publicidad y restricción a los anuncios de cigarros y de promoción de la salud, sí llegan a impactar a las adolescentes, sobre todo si los mensajes se orientan a lograr la predisposición individual contra el cigarro.

Tanto para los estudiantes de nivel medio superior como para los de licenciatura, se observó un brusco incremento del consumo a partir de 1993, año en el que la inversión en publicidad de las tabacaleras en México se duplicó respecto de 1989 y a partir de esa fecha cada año la cantidad de dinero orientado a atraer hacia el consumo a gente joven, particularmente mu- 
jeres, no ha cesado de crecer hasta llegar a cifras que en 1996 rebasaron los 3000 millones de pesos invertidos en publicidad, lo que equivale a destinar a ese fin 28 centavos de cada peso obtenido por ventas de tabaco.*

Entre los predictores de consumo en la adolescencia reportados en la literatura, los más relevantes son el nivel socioeconómico, los factores ambientales, especialmente la familia y los amigos así como la disponibilidad y el acceso al tabaco, y también se reconocen factores personales y conductuales. ${ }^{33}$

Encontramos una mayor incidencia de consumo entre adolescentes de mejor posición económica, lo cual distingue a México del patrón descrito en países de ingresos altos como EUA, donde los jóvenes de menores recursos son los que más fuman. ${ }^{14}$ Por lo tanto, una de las maneras que puede ser efectiva para bloquear el acceso a los cigarros sería aplicar mayores impuestos a los productos que contienen tabaco y así desalentar el consumo. Por su edad, los estudiantes generalmente aún no son adictos y se conoce que son un grupo muy sensible a los cambios en el precio, sobre todo en los países de bajos y medianos ingresos. ${ }^{1} \mathrm{En}$ edades entre 20 y 24 años la elasticidad del precio del cigarro es mayor que entre personas más adultas, lo que quiere decir que frente al alza de los precios muchos adolescentes y jóvenes abandonan el hábito ante la imposibilidad de poder pagarlo. Lo anterior, unido a que es poco probable que quien haya llegado a los 25 años sin fumar comience a hacerlo, justifica los impuestos al tabaco como medida para frenar el consumo entre adolescentes y jóvenes. ${ }^{34}$

Se observó una incidencia de consumo durante los años de educación media superior de $20 \%$ para los hombres y de $12 \%$ para las mujeres. Por lo tanto, es necesario implantar en las escuelas de ese nivel programas de educación antitabaco y otras actividades intencionalmente diseñadas para combatir dicho hábito. Lo anterior tendría dos principales objetivos que pueden lograrse simultáneamente y de hecho se apoyan mutuamente. Primero, promover el abandono del hábito entre los alumnos que ya son fumadores y segundo, desalentar a quienes no fuman para que no se inicien. Tales programas pueden, de hecho creemos que deben, incorporarse a los planes de estudio oficiales o tomar la forma de actividades extracurriculares, pero que en el ámbito de la escuela promuevan estilos de vida más sanos. Este tipo de programas ha sido de gran

\footnotetext{
* Nielsen AC. Inversión publicitaria cigarros enero 1985-1996. México. Documento mimeo. $\mathrm{S} / \mathrm{f}$.
}

éxito en varios países europeos ${ }^{30}$ entre alumnos de secundaria y enseñanza media.

Varios trabajos muy reconocidos en el tema del tabaquismo en edades tempranas ${ }^{33,35-39}$ tratan sobre la influencia en el desempeño en la vida adulta de haber sido un adolescente fumador, para lo cual se han basado en estudios longitudinales. Sin embargo, todas las experiencias que se han llevado a cabo en México son transversales, lo anterior evidencia que hasta ahora los intentos realizados han sido aislados y que conjuntamente con las intervenciones hace falta diseñar estudios de seguimiento que permitan no sólo describir, sino comprender intrínsecamente la evolución del tabaquismo.

Detrás del análisis de la prevalencia de consumo de tabaco también aparecen diferenciales sociales que han sido bien documentados en la literatura sobre el tema. Por ejemplo, en los EUA se reporta que el consumo de cigarros en la adolescencia está asociado fuertemente con proceder de hogares de poca instrucción, con bajo rendimiento escolar y también con bajas aspiraciones académicas. ${ }^{40}$ En nuestro estudio, al analizar en diferentes años por separado (1992, 1993 y 1996) encontramos que cuando los alumnos demoran más de los tres años establecidos para cursar la educación media superior se incrementa la proporción de fumadores que entran a la universidad.

De lo anterior se desprende una pregunta, ¿fumar cigarros es en el caso de muchos adolescentes un indicador de condiciones psicosociales y económicas desventajosas existentes o es un predictor de desempeño social futuro no exitoso, o acaso ambas cosas? Para responderla se ha explorado la hipótesis de la posible 'incompatibilidad' de fumar con ciertos roles educacionales, ocupacionales y maritales. ${ }^{41}$ Por otra parte, también se ha planteado que no existe tal incompatibilidad, pero sí cierta inmadurez asociada con el fumador adolescente que le dificulta desempeñarse con éxito en esos roles. ${ }^{36}$ Cualquiera de las dos interpretaciones apunta a que el consumo de cigarros en la adolescencia puede conducir a consecuencias no deseadas en la vida adulta, más allá de lo referido estrictamente a la salud..$^{35}$ Específicamente, encontramos que se relaciona con el atraso escolar, lo cual repercutirá en el futuro.

Como se ha mencionado, la información para este estudio se obtuvo de fuentes secundarias, por lo que para responder a nuestros objetivos partimos de datos recogidos con anterioridad y que limitaron nuestras posibilidades, por ejemplo de incluir otras variables explicativas. No obstante a lo anterior, para nuestra variable dependiente siempre se recurrió a la misma pregunta, por lo que nuestros resultados son 
válidos para el periodo estudiado. Sin embargo, puede haber sesgos de información ya que el instrumento no estaba diseñado específicamente para responder a nuestros objetivos. Se ha sugerido a la Dirección General de Servicios Médicos de la UNAM, y lo han aceptado, aplicar un instrumento más elaborado para satisfacer objetivos como los que nos propusimos en este trabajo y sobre todo, que permita recoger mayor información cada año de manera estandarizada, lo cual facilitará las comparaciones y permitirá estudiar de manera más confiable la evolución del consumo de tabaco entre alumnos de nuevo ingreso.

La conclusión general que se desprende de lo observado al final del periodo de estudio es que fuma una mayor proporción de estudiantes, y además comienzan a hacerlo desde edades más tempranas; al incremento general en el consumo han contribuido notablemente las mujeres. Específicamente, el grupo de edades que cursan la educación media superior y superior son los que mayor atención requieren para prevenirlos del consumo de tabaco. Dentro de la estrategia global de control del tabaquismo la investigación debe pasar a acciones de intervención, con especial atención a la educación y a los programas de ayuda a la cesación. Bajo esta nueva perspectiva, los estudios descriptivos como éste no serán un objetivo en sí mismos, sino que deberán servir para medir la eficacia de los programas puestos en marcha. Una vez que conocemos del creciente consumo de tabaco entre los alumnos de la UNAM, el próximo paso coherente será trabajar para revertir las tendencias que se han descrito.

\section{Referencias}

1. 0 rganización Panamericana de la Salud, Banco Mundial. La epidemia del tabaquismo. Los gobiernos y los aspectos económicos del control del tabaco. O PS, W ashington, D C: 2000; Publicación Científica N 0.577.

2. Corrao MA, Emmanuel GE, Sharma N, Shokoohi DF, ed. Tobacco Control Country Profiles. Atlanta (GA):The American Cancer Society, 2000. 3. 0 rganización Panamericana de la Salud. El Tabaquismo en América Latina, Estados Unidos y Canadá (periodo 1990-1999). W ashington DC: División de Promoción y Protección de la Salud (HPP) Programa de Prevención y Control del U so del Tabaco, Junio 2000.

4. N elson DE, Giovino GA, Shopland DR, Mowery PD, Mills SL, Ericksen MP.Trends in cigarette smoking among US adolescents, 1974 through 1991. Am J Public Health 1995;85(1):34-40.

5. United States D epartment of Health and Human Services:Tobacco use among US racial/ethnic minority group:A report of the Surgeon General. Atlanta (GA): U.S. D epartment of Health and Human Services, Centers for Disease Control and Prevention, $\mathrm{N}$ ational Center for Chronic Disease Prevention and Health Promotion, $\mathrm{O}$ ffice on smoking and Health, 1998.
6. Centers for D isease Control and Prevention. Cigarette smoking among youth-United States, 1989. MMW R Morb Mortal W kly Rep 1991; 40(41): 712-715.

7. Centers for Disease Control and Prevention. Tobacco use among high school students-United States, 1990. MMW R Morb Mortal W kly Rep 1991;40(36):617-619.

8. Centers for Disease Control and Prevention. Tobacco use and usual source of cigarettes among high school students-United States, 1995. MMW R Morb Mortal W kly Rep 1996;45(20):413-418.

9. Centers for Disease Control and Prevention. Tobacco use among high school students-United States, 1997. MMW R Morb Mortal W kly Rep 1998;47(12):229-233.

10. Centers for D isease Control and Prevention. Cigarette smoking among high school students youth-11 States, 1991-1997. MMW R Morb Mortal W kly Rep 1999;48(31):686-692.

11. Giovino GA, Schooley MW, Zhu B-P, Chrismon JH, Tomar SL, Peddicord JP et al. Surveillance for selected to bacco-use behaviors-U nited States, 1990-1994. MMW R Morb Mortal W kly Rep 1994;43(SS-3):1-43.

12. Kann L, W arren CW, Harris W A, Collins JL, W illiams BI, Ross GJ et al. Youth risk behaviour surveillance-U nited States, 1995. MMW R Morb Mortal W kly Rep 1996;45(SS-4):1-83.

13. Kessler DA,W itt AM, Barnett PS, Zeller MR, $N$ atanblut SL,W ilkenfeld JP et al. The Food and Drug Administration's regulation of tobacco products. N Engl J Med 1996;335:988-994.

14. U nited States D epartment of Health and Human Services: Preventing tobacco use among young people:A report of the Surgeon General. Atlanta (GA): US. D epartment of Health and Human Services, Centers for Disease Control and Prevention, National Center for Chronic Disease Prevention and Health Promotion, $\mathrm{O}$ ffice on smoking and Health, 1994.

15. Evans N, Farkas A, Gilpin E, Berry C, Pierce JP. Influence of tobacco marketing and exposure to smokers on adolescent susceptibility to smoking. J N al C ancer Inst 1995;87(20):1538-1545.

16. French SA, Perry CL, Leon GR, Fulkerson JA.W eight concerns, dieting behaviour, and smoking initiation among adolescents:A prospective study. Am J Public Health 1994;84(811):1818-1820.

17. Brownson RC, Jackson-Thompson J, W ilkerson JC, D avis JR, 0 wens N W, Fisher EB. D emographic and socio-economic differences in beliefs about the health effects of smoking. Am J Public Health 1992;82(1): 99-103.

18. Tomar SL, Giovino GA, Eriksen MP. Smokeless tobacco brand preference and brand switching among US adolescents and young adults. Tobacco Control 1995;4(1):67-72.

19. Secretaría de Salud. Encuesta N acional sobre el Uso de Drogas en la Comunidad Escolar. México. México, D.F.: Instituto N acional de Psiquiatría, Secretaría de Salud, 1992.

20. Castro ME, Maya MA, Aguilar MA. Consumo de sustancias tóxicas y tabaco entre la población estudiantil de 14 a 18 años. Salud Publica Mex 1982;24(5):565-574.

21. Berenson S, Medina-Mora ME, C arreño F, Juárez F,Villatoro J, Rojas E et al. Encuesta sobre el consumo de drogas en la comunidad escolar. Tendencias del consumo de sustancias psicoactivas entre estudiantes de enseñanza media y media superior del Distrito Federal. Salud Mental 1996;19(1):1-5.

22.Villatoro JA, Medina-Mora ME, Cardiel H, Fleiz C,Alcántar E, Hernández $\mathrm{S}$ et al. La situación del consumo de sustancias entre estudiantes de la Ciudad de México. Salud Mental 1999;22(2):18-30.

23. Tapia R, Lazcano F, Herrera M, Sepúlveda J. El consumo de tabaco en México. Resultados de la Encuesta N acional de Salud. Boletín Mensual de Epidemiología. 1989;4(3):33-39.

24.Tapia R, Medina ME, Sepúlveda J, De la Fuente R, Kumate J. La Encuesta N acional de Adicciones de México. Salud Publica Mex 1990;32:507-522. 25. Secretaría de Salud. Encuesta N acional de Adicciones II. 4 tomos. México, D.F.: Dirección General de Epidemiología, 1994. 
26. Kuri P, ed. N ational Addiction Survey. México, D.F.: IN EGI, SSA, D irección General de Epidemiología, 1998.

27. Gordis, L. Epidemiology. Filadelfia:W B Saunders Company, 1996.

28. Centers for D isease Control and Prevention Illegal sales of cigarettes to minors-Mexico City, 1997. MMW R Morb MortalW kly Rep 1997;46(20): 440-444.

29. Ley General de Salud, fracción II, artículo 188, publicada en el Diario 0 ficial de la Federación el 31 de mayo de 2000.

30. Savolainen S, ed.The smoker-free class competition.A European schoolbased anti-smoking campaign. An overview of the 1997/98 round in seven countries. Helsinki: $\mathrm{N}$ ational Public Health Institute, D epartment of Epidemiology and Health Promotion European N etwork on Young People and Tobacco, 1998

31. W akefield MA, Chaloupka FJ, Kaufman N J, 0 rleans CT, Barker DC, Ruel EE. D o restrictions on smoking at home, at school and public places influence teenage smoking?A cross sectional study.Trabajo presentado en The 11 W orld Conference on Tobacco or Health; 2000 August $6-11 ;$ C hicago (III), EUA.

32. Pierce JP, Lee L, Gilpin EA. Smoking initiation by adolescents girls, 1944 through 1988. JAMA 1994;271(8):608-611.

33. Conrad KM, Flay BR, Hill D. W hy children start smoking cigarettes: Predictors of onset. Br J Addict 1992;87:697-706.

34. Lewit EM.The effects of government regulation on teenage smoking.J Law Econ 1981;24:545-575.
35. Chassin L, Presson CC, Sherman SJ, Edwards DA. The natural history of cigarettes smoking and young adult social roles. J Health Soc Behav 1992;33(4):328-347.

36. N ewcomb MD, Bentler PM. Consequences of adolescents drug use: Impact on the life of young adults. N ewbury Park: Sage Publication, 1988. 37. Chassin L, Presson CC, Sherman SJ, Edwards DA. The natural history of cigarettes smoking: Predicting young adult smoking outcomes from adolescent smoking patterns. Health Psychol 1990;9:703-718.

38. Paavola M,Vartiainen E, Puska P. Predicting adult smoking:The influence of smoking during adolescence and smoking among friends and family. Health Educ Res 1996;11(3):309-315.

39.Vartiainen E, Paavola M, M CA lister A, Puska, P. Fifteen-year follow-up of smoking prevention effects in the N orth Karelia Youth Project. Am J Public Health 1998;88:81-85.

40. United States Surgeon General. Reducing the health consequences of smoking: 25 years of progress.W ashington, DC:G overnment Printing 0 ffice, 1989.

41.Yamaguchi K, Kandel D B. $0 \mathrm{n}$ the resolution of role incompatibility: A life events analysis of family roles and marijuana use. Am J Sociol 1985; 90:1284-1325. 